\title{
Aprender de lo sucedido. Análisis de las quejas presentadas ante la Comisión Nacional de Arbitraje Médico
}

\author{
Raydel Valdés-Salgado, MC, ${ }^{1}$ Joaquín Molina-Leza, $\mathrm{Dr},{ }^{2}$ \\ Cuauhtémoc Solís-Torres, Lic. ${ }^{3}$
}

Valdés-Salgado R, Molina-Leza J, Solís-Torres C. Aprender de lo sucedido. Análisis de las quejas presentadas ante la Comisión Nacional de Arbitraje Médico. Salud Publica Mex 2001;43:444-454.

El texto completo en inglés de este artículo está disponible en: http://www.insp.mx/salud/index.html

\section{Resumen}

Objetivos. Describir las quejas por insatisfacción con los servicios médicos que se presentaron ante la Comisión $\mathrm{N}$ acional de Arbitraje Médico (Conamed), en 1997 y 1998, procedentes de México, D istrito Federal.También, conocer qué aspectos de la calidad de la atención aparecen como deficientes, según las instituciones, las especialidades y algunas características de los quejosos. Material y métodos. Se estudiaron 1925 quejas y se sistematizó la información para su análisis cuantitativo. Se aplicó la prueba ji cuadrada $\left(\chi^{2}\right)$ de Pear son para muestras independientes y un análisis de conglo merados. Resultados. Las quejas, principalmente, son relativas al tratamiento, las intervenciones quirúrgicas y el diagnóstico. Para cada una de las instituciones involucradas se distingue si las quejas se refieren a la estructura, al proceso 0 al resultado de la atención. Los tipos de conflictos difieren según el sexo, la edad y nivel socioeconómico de las personas. Conclusiones. Las quejas llevadas ante la Conamed aportan elementos sobre la satisfacción de los usuarios y la calidad de la atención; nuestros resultados son consistentes con los resultados de otros trabajos previos que han estudiado la satisfacción de los usuarios y la calidad de la atención. El texto completo en inglés de este artículo está disponible en: http://www.insp.mx/salud/index.html

Palabras clave: quejas; insatisfacción de los usuarios; calidad de la atención; México

\author{
Valdés-Salgado R, MolinaLeza J, Solís-Torres C. \\ Learning from experience. \\ User's dissatisfaction \\ with health services through claims brought to Conamed \\ Salud Publica Mex 2001;43:444-454. \\ The English version of this paper \\ is available at: http://www.insp.mx/salud/index.html
}

\begin{abstract}
A bstract
Objectives To describe claims brought to Comisión $\mathrm{N}$ acional de Arbitraje Médico (Medical Arbitration N ational Commission or Conamed) against medical care services in Mexico City. Also, to identify deficiencies in the quality of care by institution and medical speciality and describe some characteristics of the plaintiffs. Material and Methods A total of 1925 claims were collected and data were analysed quantitatively. Pearson's chi-square (c2) test and a cluster analysis were performed. Results. Most claims are related to therapy, surgery, and diagnosis problems. Claims related to structure, process, and outcomes were also identified for each defendant institution. Claims vary by sex, age, and socio-economic levels. Conclusions. Claims brought to $\mathrm{Co}$ named are useful indicators of users' dissatisfaction. 0 ur results are consistent with other papers about users' satisfaction and quality of health care.The English version of this paper is available at: http://www.insp.mx/salud/index.html
\end{abstract}

Key words: claims; user's dissatisfaction; quality of health care; Mexico

N ota: este artículo expone parcialmente el contenido de la tesis "Satisfacción de los U suarios de Servicios de Salud en México, D.F.A nálisis de las demandas presentadas a la Conamed", que para optar por el grado de Maestro en Población, con especialización en Población y Salud, se presentó el 3 de agosto de 1999 en la Facultad Latinoamericana de Ciencias Sociales (Flacso), Sede Académica de México.

(1) Centro de Investigación en Salud Poblacional, Instituto N acional de Salud Pública, México.

(2) Sistemas y Servicios de Salud de la Representación en México, 0 rganización Panamericana de la Salud, oficina regional de la 0 rganización Mundial de la Salud.

(3) Dirección General de Investigación y Métodos de la Comisión N acional de Arbitraje Médico, 1996-2000.

Fecha de recibido: 7 de abril de 2000 - Fecha de aprobado: 11 de junio de 2001

Solicitud de sobretiros: M. Raydel Valdés Salgado. Centro de Investigación en Salud Poblacional, Instituto N acional de Salud Pública. Avenida Universidad, colonia Santa María A huacatitlán, 62508 Cuernavaca, Morelos, México.

Correo electrónico: rayvs@ correo.insp.mx 
C uando se asume la responsabilidad de analizar información planteada originalmente como quejas, hay que ser conscientes de las cinco acepciones aceptadas ${ }^{1}$ para ese término: 1 ) expresión de dolor; 2) manifestación de disconformidad, disgusto o descontento; 3) motivo de queja; 4) recurso a interponer por la parte interesada en contra de algo y 5) querella. Lo anterior implica que el investigador adopte una postura ética, que lo lleve a sentir respeto por las partes involucradas en cada caso; el manejo responsable de la información disponible, y sobre todo, la intención de mostrar con los resultados de su estudio los problemas subyacentes, de manera tal que al identificarlos se eviten situaciones futuras que causen daños a más personas. Todo esto cobra mayor relevancia cuando se trata de quejas que se originaron en los servicios médicos.

Se acepta que la satisfacción de los usuarios constituye una medición útil de la calidad de las consultas y de la relación médico-paciente. ${ }^{2}$ Asimismo, las estimaciones basadas en la satisfacción de los pacientes son un indicador de la ejecución del sistema y permiten diseñar diferentes estrategias alternativas para ofrecer los servicios. ${ }^{3}$ La valoración de la calidad de los servicios a partir de las quejas de los usuarios de servicios médicos, es coherente dentro del modelo de la discrepancia de Williams, ${ }^{4}$ donde se le concede a las expectativas del paciente un papel fundamental para explicar la satisfacción o insatisfacción reportada.

Los estudios sobre calidad enfrentan el problema de la ambigüedad del concepto. Una de las definiciones más aceptadas, aplicada específicamente a los servicios de salud, es la propuesta por Avedís Donabedian. " "La calidad de la atención a la salud es el grado en que los medios más deseables se utilizan para alcanzar las mayores mejoras posibles en la salud. Sin embargo, como las consecuencias de la atención se manifiestan en un futuro que frecuentemente resulta difícil de conocer, lo que se juzgan son las expectativas de resultado que se podrian atribuir a la atención en el presente."

Este trabajo se apoya en la concepción de Donabedian, quien señaló ${ }^{6}$ que independiente de la amplitud de la definición de calidad, en cualquier programa o servicio se pueden evaluar la estructura, el proceso y los resultados. En nuestro estudio se hizo una clasificación de las quejas según esta aproximación.

El término estructura menciona todos los atributos -materiales y organizacionales- relativamente estables en los sitios en donde se proporciona la atención. El proceso incluye todo lo que médicos y otros proveedores de atención hacen por los pacientes, así como la habilidad con la cual lo llevan a cabo. Finalmente, el término resultados comprende lo que se ha obtenido para el paciente; específicamente supone un cambio en el estado de salud, para bien o para mal, e incluye otras consecuencias de la atención, por ejemplo el conocimiento adquirido sobre la enfermedad, y cambios en la conducta que conducen a la salud y la satisfacción del paciente.

Donabedian ${ }^{7}$ describió la calidad de la atención en tres dimensiones: la técnica, la interpersonal y la de las comodidades. La primera entendida como la aplicación de la ciencia y la tecnología a la solución del problema de salud del paciente. La segunda se expresa en la relación que se establece entre prestadores y receptores de los servicios. Por último, las comodidades son todos los elementos del ambiente físico que rodean el proceso de atención.

Por lo general, la cuestión técnica predomina en las valoraciones de los prestadores, mientras que los usuarios conceden más atención a la relación interpersonal y las comodidades. Los resultados de Hall y colaboradores ${ }^{8}$ confirman esta discrepancia.

La bibliografía de finales de los años 80 e inicio de los 90 refleja lo que se ha denominado la perspectiva del consumidor. ${ }^{9,10}$ El interés por conocer la satisfacción de los usuarios responde a la concepción del receptor de los servicios como el árbitro final de la calidad. ${ }^{11}$ Si bien lo anterior comenzó en sistemas de salud distintos a los latinoamericanos, incluido el mexicano, por supuesto, más recientemente la idea de que la garantía de la atención está dada por la satisfacción del cliente ${ }^{12}$ está implícita en no pocos procesos nacionales de reforma del sector salud iniciados en la región.

Tradicionalmente se ha reconocido como importante la opinión de los pacientes, sin embargo, en los estudios realizados, predomina la de los profesionales de la salud. ${ }^{13}$ Lo anterior está muy marcado por la idea de que los pacientes son incapaces de emitir un juicio imparcial y, por tanto, su criterio es subjetivo. ${ }^{14}$

En general, a las investigaciones diseñadas para estudiar la satisfacción de los pacientes con la calidad de la atención recibida, se les señala que sus resultados están influenciados por el método de recolección de la información y los instrumentos empleados, recomendándose cada vez más las aproximaciones cualitativas. ${ }^{15}$ No existe consenso sobre qué aspectos son los que evalúan las personas. En un reconocido meta-análisis, Hall y Dorman ${ }^{16}$ revisaron 221 trabajos previos y reportan doce elementos-categoría referidos a las dimensiones de la satisfacción de los pacientes.

Al preguntar a los usuarios por la calidad en general, se ha encontrado un elevado por ciento de satisfacción, sin embargo, con preguntas más específicas se encuentran evidencias de insatisfacción. ${ }^{17}$ Esto fue 
corroborado por Carr-Hill y colaboradores ${ }^{18}$ al preguntar sobre situaciones particulares como describir la última visita al médico. En general, las personas distinguen entre los aspectos operativos y los afectivos de la atención recibida, ${ }^{2,19}$ y también se ha informado que las personas se muestran ambivalentes y escépticas ante los avances de la medicina moderna. ${ }^{20}$

En este trabajo se analizan datos derivados directamente de la información expresada de forma espontánea por los usuarios que se quejaron de los servicios de salud. Se recurre a una forma no convencional de abordar el tema de la insatisfacción de los pacientes, que es el estudio de todas las quejas llevadas ante la Comisión Nacional de Arbitraje Médico, Conamed, durante 1997 y 1998 procedentes del Distrito Federal. Esto nos permitió identificar aspectos que, desde la perspectiva de los usuarios quejosos, constituyen deficiencias en la calidad de la atención médica. Es oportuno destacar que en todo el trabajo se usa la palabra 'quejoso' para señalar a la persona que plantea la queja; no es en lo absoluto un término peyorativo, sino que así es como se refieren en la Conamed y nos pidieron que conserváramos la expresión.

Estudiar las quejas presentadas ante la Conamed nos parece muy oportuno porque en México existe el antecedente de que la negligencia médica y la negativa de atención están anualmente entre las más frecuentes presuntas violaciones al derecho a la protección de la salud presentadas en la Comisión Nacional de Derechos Humanos, ${ }^{21-25}$ lo anterior sin considerar los casos en donde se involucra al sector privado. El número de quejas que vincula a cada institución en el presente estudio, suponemos refleja un subregistro de las inconformidades de la población, pues la Conamed, y las posibilidades que ésta ofrece, son aún poco difundidas entre la población.

\section{¿Qué es la Comisión Nacional de Arbitraje Médico?}

La Conamed es un órgano desconcentrado de la Secretaría de Salud creado en junio de 1996, con plena autonomía técnica para emitir sus opiniones, acuerdos y laudos. Su objeto es la resolución de conflictos suscitados entre los usuarios de los servicios médicos y los prestadores de servicios de salud de carácter público, privado y social, así como contribuir a mejorar la calidad y eficiencia de los servicios médicos en México. ${ }^{26}$ Las quejas atendidas en la Comisión son exclusivamente aquellas en las cuales las partes involucradas coinciden en someter ante esta instancia su valoración.

La población puede acudir voluntariamente a la Conamed a plantear una queja por insatisfacción con la atención médica recibida. En la Comisión se solucionan controversias civiles por la vía de la asesoría e información, la conciliación y el arbitraje. ${ }^{27}$ Los casos deben argumentar y documentar suficientemente su queja para que sea aceptada y de inmediato la Comisión notifique a los prestadores involucrados. En todo el proceso siguiente, el análisis del expediente clínico desempeña un papel fundamental.

Una característica del trabajo de la Conamed es la inclusión de la perspectiva del usuario en la descripción de los hechos y el planteamiento de pretensiones, es decir, lo que la persona pide a manera de solución (cuyo análisis ya hemos publicado). ${ }^{28}$ Esto permite que se cuente con una descripción espontánea de los problemas que los usuarios reportan como deficiencias de la calidad del servicio médico recibido. Otro rasgo distintivo es que la información se recoge fuera de un ambiente médico, gracias a ello las personas se pueden sentir libres para expresar sus inconformidades.

La población que acude a la Conamed siempre plantea situaciones en las que presumiblemente se cometió algún tipo de negligencia, o la parte quejosa considera que el comportamiento y desempeño de los prestadores de servicios médicos involucrados es cuestionable. Por lo anterior, todos los casos plantean esencialmente insatisfacción e, implícitamente, en sus asuntos se identifican elementos de la calidad de la atención recibida. Esta información es valiosa por la manera espontánea en que es expresada originalmente, y por lo tanto, a través de ella se puede saber quiénes se quejan, de qué se queja la población y qué instituciones están involucradas y por qué.

Aquí presentamos una parte de los resultados de una investigación empírica que buscó responder las preguntas planteadas anteriormente. A manera de respuestas encontramos asociaciones significativas que se describen en detalles más adelante. Con independencia de que la información analizada no se obtiene de un protocolo diseñado específicamente para estudiar calidad de la atención o satisfacción de los usuarios, consideramos que las quejas ante la Conamed aportan elementos relevantes en ambos sentidos.

En este artículo sólo se analizan las quejas que llegan a la Conamed y no el desempeño de esa institución, lo cual es tema de otro trabajo de los mismos autores, aún no publicado.

\section{Material y métodos}

En este trabajo se analizan todas las quejas procedentes del Distrito Federal presentadas ante la Conamed en el periodo comprendido entre el $1^{\circ}$ de enero de 1997 y el 31 de diciembre de 1998. Se trata de 1925 casos 
que acudieron voluntariamente; 893 quejas corresponden a 1997 y las restantes 1032 se presentaron en 1998. De los casos, 1184 son mujeres y 730 hombres, en otros 11 no se registró el sexo. Las edades varían desde los 2 días de nacido hasta los 95 años. El promedio de edad de las mujeres, $41.81 \pm 18.83$ años, es similar al de los hombres 41.22 \pm 22.04 . En ninguno de los casos se tuvo acceso a información referida a la identidad de las partes involucradas.

Se trabajó con la información procedente del Distrito Federal, por ser la entidad federativa de mayor concentración de recursos de salud del país y por distinguirse de las restantes en que aporta el mayor número de quejas, ofreciendo mejores posibilidades para la interpretación estadística de los resultados. En el periodo estudiado existían sólo dos comisiones estatales de arbitraje médico en el interior del país, por ello, probablemente, gran parte de la población que reside fuera de la capital desconocía la Conamed.

En el momento en que la queja es registrada, ésta se clasifica según criterios propios de la Comisión, considerando para ello la descripción detallada de los hechos. Se manejan siete tipos de quejas relacionadas con diagnóstico, tratamiento, auxiliares de diagnóstico y tratamiento, intervenciones quirúrgicas, hospitalización, relación médico-paciente y otras quejas. Además de esa clasificación general, se recogen otros detalles de las circunstancias de la queja mismos en los que nos basamos para clasificarlas según la propuesta de Donabedian, ${ }^{6}$ considerando si se relacionan con la estructura, el proceso o los resultados de la atención.

Las quejas analizadas estaban referidas a 49 especialidades y servicios médicos. Esta dispersión dificultaba la interpretación de los resultados e impedía la aplicación de pruebas de significación estadística. Las especialidades fueron reagrupadas en categorías construidas ad hoc que se muestran en el cuadro I, para cuya clasificación nos basamos en el criterio de jueces. ${ }^{29}$ En los casos en que no hubo coincidencia, se llegó al consenso mediante discusión.

Respecto a las personas quejosas sólo se disponía del sexo y la edad. Para clasificarlas según el nivel socioeconómico se hizo una inferencia ecológica a partir de las colonias de residencia. Para ello nos apoyamos en una clasificación prexistente ${ }^{30}$ de estratificación unívoca y exhaustiva, donde cada colonia urbana de México pertenece a uno de seis niveles propuestos, considerando los bienes durables que poseen los hogares, las características de las viviendas y otros aspectos socioculturales. La clasificación por nivel socioeconómico nos parece más útil que otras, tales como ase- gurados o no asegurados, porque comprobamos que en cada caso pueden aparecer involucradas diferentes tipos de instituciones, y uno de nuestros resultados es, precisamente, que muchos derechohabientes combinan la atención de la seguridad social y del sector privado, o simplemente acuden a este último. Por lo tanto, son en última instancia las condiciones socioeconómicas las que inciden en el tipo de institución a la que se acude.

Según la clasificación empleada, al nivel socioeconómico más pobre pertenecen las familias que reciben mensualmente $\$ 1500$ o menos. En orden ascendente siguen los que ganan hasta $\$ 3000$; después las familias con ingresos de entre $\$ 4000$ y $\$ 5000$. Los que denominamos clase media, ganan entre $\$ 6000 \mathrm{y}$ $\$ 20$ 000. La clase alta son familias que reciben entre $\$ 21000$ y $\$ 50000$ y más de $\$ 51000$.

Con base en las definiciones de A. Donabedian, ${ }^{6}$ se analizó cada caso y se hizo una clasificación según si se referían a la estructura, el proceso o los resultados de la atención, la misma es resultado del criterio de jueces expertos que analizaron las variables tipo y clase de queja, y cuando no hubo coincidencia, se llegó al consenso mediante discusión. Más detalles se pueden revisar en la investigación más amplia de la cual se derivan estos resultados. ${ }^{29}$

Se consideraron problemas en la estructura del servicio 187 quejas como las siguientes: falta de equipos e insumos, resultados inoportunos, todo tipo de diferimientos injustificados, la falta del personal necesario y la falta de notificación.

Las quejas clasificadas como problemas en el proceso de atención fueron 1324 y se relacionan con diagnósticos erróneos, innecesarios y/o inoportunos; tratamientos inadecuados, inoportunos, insatisfactorios y / o innecesarios; todo tipo de resultados falso positivo y falso negativo; impericia y empleo de técnica inadecuada en la intervención quirúrgica. También, las cirugías innecesarias, las extirpaciones erróneas, así como los accidentes e incidentes durante la intervención quirúrgica.

Asimismo, se consideraron los casos de hospitalización prolongada y de atención inoportuna durante la hospitalización, así como la relación médico-paciente caracterizada por la desinformación o dar información errónea, malos tratos y negación del servicio, abusos y las acciones fuera de norma.

Las quejas que indican problemas con los resultados de la atención fueron 405 y se refieren a situaciones como complicaciones secundarias, secuelas y complicaciones posquirúrgicas. También los resultados no satisfactorios y las complicaciones y enfermedades secundarias en relación con hospitalizaciones. 


\section{Cuadro I}

Agrupamientos de las quejas según SeRvicios y especialidades médicas.

Comisión Nacional de Arbitraje Médico. México 1997-1998

\begin{tabular}{|c|c|c|c|}
\hline Especialidades/servicios & Quejas & Etiqueta & Casos \\
\hline Ortopedia & 246 & Traumatología y ortopedia & 282 \\
\hline Traumatología & 36 & & \\
\hline Anestesiología & 18 & Asociadas a actos quirúrgicos & 246 \\
\hline Cirugía general & 137 & & \\
\hline Cirugía plástica & 40 & & \\
\hline Cirugía pediátrica & 4 & & \\
\hline Cirugía reconstructiva & 5 & & \\
\hline Neurocirugía & 32 & & \\
\hline Cirugía & 10 & & \\
\hline Medicina familiar & 140 & Servicios médicos generales & 235 \\
\hline Medicina general & 26 & & \\
\hline Medicina interna & 61 & & \\
\hline Medicina preventiva & 3 & & \\
\hline Medicina del trabajo & 5 & & \\
\hline Urgencias & 160 & & \\
\hline 0 dontología & 147 & Salud bucal & 148 \\
\hline Estomatología & 1 & & \\
\hline Audiología y fonotría & 1 & Organos de los sentidos y piel & 141 \\
\hline Dematología & 11 & & \\
\hline Oftalmología & 94 & & \\
\hline Otorrinolaringología & 35 & & \\
\hline O bstetricia & 128 & Salud reproductiva & 134 \\
\hline Planificación familiar & 6 & & \\
\hline Ginecología & 124 & Ginecología & 124 \\
\hline $\begin{array}{l}\text { Endocrinología } \\
\text { Gastroenterología }\end{array}$ & $\begin{array}{r}8 \\
35\end{array}$ & 0 tras especialidades & 131 \\
\hline Hematología & 8 & & \\
\hline Neumología & 9 & & \\
\hline Neurofisiología & 1 & & \\
\hline Neurología & 38 & & \\
\hline Proctología & 9 & & \\
\hline Psicología & 3 & & \\
\hline Psiquiatría & 20 & & \\
\hline N efrología & 27 & Riñón y vías urinarias & 84 \\
\hline Urología & 57 & & \\
\hline Angiología & 21 & Cardiovascular & 70 \\
\hline Cardiología & 49 & & \\
\hline N eonatología & 4 & Población infantil & 63 \\
\hline Pediatría & 49 & & \\
\hline Perinatología & 10 & & \\
\hline O ncología & 52 & O ncología & 52 \\
\hline Endoscopía & 2 & A poyo al diagnóstico y tratamiento & 34 \\
\hline Enfermería & 3 & & \\
\hline Imagenología & 2 & & \\
\hline Radiología & 5 & & \\
\hline Rehabilitación y fisioterapia & 6 & & \\
\hline \multirow[t]{2}{*}{ Terapia intensiva } & 7 & & \\
\hline & 9 & & \\
\hline Administración & 17 & Administrativas & 17 \\
\hline Especialidad no definida & 4 & No definidas & 4 \\
\hline Total & 1925 & & 1925 \\
\hline
\end{tabular}




\section{A nálisis estadístico}

Se aplicó la prueba de ji cuadrada $\left(\chi^{2}\right)$ para determinar la significancia de las asociaciones observadas entre grupos independientes. ${ }^{31}$ Las variables sometidas a este procedimiento están definidas en la escala nominal y otras en la escala ordinal.

Los asuntos presentados a la Conamed se clasificaron mediante un análisis de conglomerados ${ }^{32}$ según las características de las personas, tipo de quejas y las instituciones. Después de siete iteraciones se obtuvieron los agrupamientos a partir de las similaridades (distancias) entre los casos. Se empleó el método no jerárquico de las $k$ medias, que asigna cada caso al conglomerado que tenga el centroide (media) más próximo a su valor. Para todos los procesamientos de la información se usó el paquete estadístico SPSS para Windows, versión 9.0.1 (1999).

\section{Resultados}

A continuación se describen las quejas analizadas, considerando las instituciones y los tipos de quejas, así como algunas características de las personas afectadas. Las instituciones involucradas se encuentran en el cuadro II. En orden decreciente de frecuencia aparecen el Instituto Mexicano del Seguro Social (IMSS), los servicios privados, el Instituto de Seguridad y Servicios Sociales de los Trabajadores del Estado (ISSSTE), las Instituciones para población no asegurada de la Secretaría de Salud y otros servicios públicos; asimismo, instituciones como los servicios médicos de la empresa Petróleos Mexicanos, la Secretaría de la Defensa Nacional y la Secretaría de Marina.

En el cuadro III se muestra la distribución de frecuencias del tipo de quejas. Los asuntos relacionados con el tratamiento prescrito, los originados por insatisfacción con las intervenciones quirúrgicas y los asociados con el diagnóstico emitido representan $83.5 \%$ del total.

$\mathrm{Al}$ analizar las quejas y las instituciones involucradas se halló el siguiente patrón significativo $\left(\chi^{2}=\right.$ $118.0780 \operatorname{con} p=0.000)$; antes de presentar estas asociaciones se descartó el posible efecto de interacción que pudieran haber tenido el sexo y el nivel socioeconómico de los quejosos.

Las quejas por insatisfacción con el diagnóstico se asocian fundamentalmente a las unidades del IMSS y el ISSSTE; mientras tanto la insatisfacción con el tratamiento aparece generalmente relacionada con las quejas que involucran servicios privados. Los casos que tienen que ver con intervenciones quirúrgicas se asocian mayormente con las instituciones que atienden

\section{Cuadro II \\ Quejas ReCiBidas DE CADA INSTITUCIÓN. MÉxıco 1997-1998}

\begin{tabular}{lc} 
Institución demandada & Quejas recibidas \\
Instituto Mexicano del Seguro Social & 775 \\
\hline & $(40.2 \%)$ \\
Sector privado & 520 \\
\hline & $(27.0 \%)$ \\
Instituto de Seguridad y Servicios Sociales & \\
para los Trabajadores del Estado & 462 \\
\hline & $(24.0 \%)$ \\
Instituciones para población no asegurada & 143 \\
\hline & $(7.4 \%)$ \\
O tras instituciones & 25 \\
\hline & $(1.2 \%)$ \\
\hline Total & 1925 \\
\hline
\end{tabular}

Fuente: Estimaciones propias en base a datos de la Comisión $\mathrm{N}$ acional de Arbitraje Médico

población no asegurada y con el sector privado. Aunque el número de quejas sobre la relación médicopaciente no es muy elevado, fundamentalmente se asocia a las unidades del IMSS y a las instituciones que atienden población no asegurada. La categoría Otras quejas, que se refiere fundamentalmente a problemas de tipo administrativo, se vincula más con las unidades del IMSS y el ISSSTE.

Hay asociación entre las variables tipo de quejas, grupos de especialidades médicas y las instituciones.

Los casos relacionados con problemas de diagnóstico se asocian significativamente con los servicios de urgencias y los servicios médicos generales. También, aunque en menor medida, a los servicios que atienden población infantil, oncología y otras especialidades $\left(\chi^{2}=\right.$ 186.6754 con $p=0.000$ ). Las unidades del ISSSTE son las que aparecen más asociadas con las quejas de diagnóstico $\left(\chi^{2}=57.4050 \operatorname{con} p=0.000\right)$.

Las inconformidades relacionadas con el tratamiento están asociadas, principalmente, con salud bucal, con urgencias, con las especialidades que atienden población infantil y con las que se denominaron otras especialidades $\left(\chi^{2}=254.8652\right.$ con $\left.p=0.000\right)$.

El análisis por instituciones evidenció que este tipo de queja se asocia con las unidades del IMSS, en par- 


\section{Cuadro III \\ Clasificación de las quejas presentadas ante la Comisión Nacional de Arbitraje Médico. México 1997-1998}

\begin{tabular}{|c|c|c|c|}
\hline \multirow[b]{2}{*}{ Quejas relacionadas con } & \multirow[b]{2}{*}{ Total } & \multicolumn{2}{|c|}{ Por sexo } \\
\hline & & Mujeres & Hombres \\
\hline \multirow[t]{2}{*}{ Tratamiento } & 719 & & \\
\hline & $37.4 \%$ & $38.5 \%$ & $35.3 \%$ \\
\hline \multirow[t]{2}{*}{ Intervenciones quirúrgicas } & 556 & & \\
\hline & $29.8 \%$ & $30.2 \%$ & $26.5 \%$ \\
\hline \multirow[t]{2}{*}{ Diagnóstico } & 331 & & \\
\hline & $17.2 \%$ & $16.4 \%$ & $18.4 \%$ \\
\hline \multirow[t]{2}{*}{0 tras quejas } & 99 & & \\
\hline & $5.1 \%$ & $4.6 \%$ & $5.8 \%$ \\
\hline \multirow[t]{2}{*}{ Relación médico-paciente } & 93 & & \\
\hline & $4.8 \%$ & $4.5 \%$ & $5.3 \%$ \\
\hline \multirow[t]{2}{*}{ Auxiliares de diagnóstico y tratamiento } & 68 & & \\
\hline & $3.5 \%$ & $2.8 \%$ & $3.2 \%$ \\
\hline \multirow[t]{2}{*}{ Hospitalización } & 59 & & \\
\hline & $3.1 \%$ & $2.6 \%$ & $5.0 \%$ \\
\hline \multirow[t]{2}{*}{ Total } & 1925 & & \\
\hline & $100.0 \%$ & $100.0 \%$ & $100.0 \%$ \\
\hline
\end{tabular}

Fuente: Estimaciones propias en base a datos de la Comisión $\mathrm{N}$ acional de Arbitraje Médico

ticular con las especialidades que sirven de apoyo al diagnóstico y tratamiento y con las que atienden los órganos de los sentidos y la piel $\left(\chi^{2}=37.0018\right.$ con $p=$ 0.00074).

Las quejas relacionadas con intervenciones quirúrgicas tienen asociación fundamentalmente con traumatología y ortopedia y, como era de esperar, con todo lo relacionado con actos quirúrgicos. También con ginecología y las especialidades de los órganos de los sentidos o la piel, así como con lo referido a la salud reproductiva $\left(\chi^{2}=488.4719 \operatorname{con} p=0.000\right)$. Controlando por instituciones, la mayor asociación se encuentra con las unidades del IMSS, no sólo en los servicios ya citados, sino también en los relacionados con el riñón y las vías urinarias $\left(\chi^{2}=170.9251\right.$ con $\left.p=0.000\right)$.

Las quejas derivadas de la relación médico-paciente se originan fundamentalmente en los servicios médicos generales y en las especialidades de traumatología y ortopedia y, aunque el número de casos es reducido, también se asocia con las especialidades de la salud reproductiva, las que atienden población infantil, oncología, ginecología y las especialidades de los órganos de los sentidos y la piel $\left(\chi^{2}=43.0142\right.$ con $p=0.00009$ ).

La categoría Otras quejas está asociada principalmente con los servicios médicos generales y con el área administrativa de las unidades de atención. También se asocia con urgencias, especialidades del riñón y de las vías urinarias, oncología, cardiovascular, con las especialidades de apoyo al diagnóstico y tratamiento, así como con el grupo de otras especialidades $\left(\chi^{2}=171.4185\right.$ con $p=0.000$ ).

Considerando las características de las quejas, éstas se clasificaron según reflejaran problemas de la estructura, del proceso o del resultado de la atención. Este criterio se aplicó a cada institución. Las asociaciones encontradas $\left(\chi^{2}=94.6249\right.$ con $\left.p=0.000\right)$ son las siguientes:

Las quejas procedentes del IMSS y el ISSSTE se asocian principalmente con problemas ocasionados por la estructura y otros ocurridos en el proceso de atención. De las instituciones que atienden población no asegurada, las quejas se asocian con problemas de estructura y también con los resultados. Por otra parte, los casos procedentes del sector privado generalmente están inconformes con los resultados.

El cuadro III muestra que los mayores porcentajes, para ambos sexos, se refieren a inconformidades relacionadas con el tratamiento, las intervenciones quirúrgicas y el diagnóstico. Sin embargo, al aplicar una prueba de ji cuadrada $\left(\chi^{2}\right)$ para dos muestras independientes se hallaron diferencias significativas $\left(\chi^{2}=\right.$ 14.2766 con $p=0.02669$ ). Las quejas de las mujeres se asocian fundamentalmente a insatisfacción con el tratamiento y con las intervenciones quirúrgicas. Por otra parte, los hombres se quejan principalmente de problemas relacionados con el diagnóstico.

Para la variable edad se halló un solo resultado significativo. Las quejas relacionadas con diagnóstico y tratamiento se asocian con los usuarios menores de 15 años -que corresponde a la atención pediátrica y, por lo tanto, estos casos siempre los presenta un familiar. También se asocian, aunque en menor medida, con las quejas sobre hospitalizaciones y con la relación médico-paciente. Por su parte, las quejas de los adultos -16 años y más- se caracterizan por asociarse, principalmente, con las intervenciones quirúrgicas $\left(\chi^{2}=\right.$ $18.3306 \operatorname{con} p=0.00546$ ).

Las quejas también se pueden distinguir por nivel socioeconómico $\left(\chi^{2}=30.1898\right.$ con $\left.p=0.03564\right)$. Los grupos más pobres se refieren fundamentalmente a asuntos relacionados con el diagnóstico y las hospita- 
lizaciones. Para los considerados clase media baja, las quejas se asocian más con las intervenciones quirúrgicas y Otras quejas, mientras que los de clase media alta se quejan principalmente en relación con el diagnóstico y la relación médico-paciente. Finalmente, los casos del grupo de mejor posición económica se refieren principalmente al tratamiento y las intervenciones quirúrgicas.

En el cuadro IV se muestra la clasificación resumida de las quejas presentadas a la Conamed, la cual se obtuvo por un análisis de conglomerados. La variable edad es la que más fuertemente discrimina entre los cuatro agrupamientos. Resulta interesante la similitud en el comportamiento que describen los conglomerados 'Niños' y 'Mujeres jóvenes', que se puede explicar porque en ambos grupos son mujeres jóvenes las que intervienen. Generalmente, es la madre quien lleva sus hijos al médico, y también son ellas quienes más frecuentemente representan a los menores en las casos planteados ante la Conamed.

Un resultado que nos parece un indicador indirecto de insatisfacción, es que los derechohabientes del IMSS y el ISSSTE buscan atención médica en otro tipo de instituciones $\left(\chi^{2}=156.0530\right.$ con $\left.p=0.000\right)$. Al revisar la descripción de hechos aparecida en las pretensiones ${ }^{28}$ de los quejosos, se conoció que la principal alternativa es el sector privado.

\section{Discusión}

Los principales motivos de queja llevados a la Conamed fueron: inconformidad con el tratamiento orientado, problemas relacionados con intervenciones quirúrgicas y con el diagnóstico emitido, todos relacionados con aspectos técnico-médicos de la atención.

Nuestros resultados acerca de lo que se quejan los usuarios de servicios médicos y las instituciones involucradas, si bien se obtienen de una forma no convencional, al abordar el tema, y además las presentan espontáneamente personas auto seleccionadas que acudieron voluntariamente a la Conamed, son consistentes con los resultados de trabajos previos diseñados específicamente para estudiar satisfacción de los usuarios y calidad de la atención. A continuación comentamos esas similitudes.

Más de la mitad de las quejas fueron planteadas por derechohabientes de la seguridad social. Lo anterior no se contradice con lo obtenido en la Encuesta Nacional de Salud II de 1994, donde los usuarios asegurados reconocieron el por ciento más bajo de buena calidad percibida. ${ }^{33}$ Los servicios privados también aparecen en nuestro estudio, involucrados, sin embargo, en un número considerable de casos en la encuesta mencionada, donde comparativamente su calidad fue la más apreciada.

\section{Cuadro IV \\ Clasificación de las demandas presentadas a la Comisión Nacional de Arbitraje Médico, SEgún Las CaRActerísticas de la QUeja y de LAS Personas afectas. México 1997-1998}

\begin{tabular}{|c|c|c|}
\hline Conglomerado & $\mathrm{n}$ & Descripción \\
\hline 1 & $\begin{array}{c}273 \\
(14.18 \%)\end{array}$ & $\begin{array}{l}\text { N iños } \\
\text { Formado por niños y niñas con edad promedio de } 9.4 \text { años, } 52 \% \text { es de clase media baja y } 31.5 \% \text { de clase media alta. } \\
\text { Las quejas se relacionan con el tratamiento ( } 41 \%) \text {, intervenciones quirúrgicas }(21 \%) \text { y el diagnóstico (20\%). } \\
\text { Generalmente fueron atendidos en unidades de hospitalización general (38\%) y en consulta externa (32\%). Proceden } \\
\text { fundamentalmente del IMSS (37.7\%) y en menor medida del sector privado (29\%). }\end{array}$ \\
\hline 2 & $\begin{array}{c}767 \\
(39.84 \%)\end{array}$ & $\begin{array}{l}\text { Mujeres jóvenes } \\
\text { Constituido fundamentalmente por mujeres (67.2\%) con un promedio de } 32.7 \text { años. Las quejas están relacionadas } \\
\text { principalmente con el tratamiento ( } 34 \% \text { ) y con las intervenciones quirúrgicas (33.6\%) y proceden de unidades de } \\
\text { hospitalización general ( } 36.2 \% \text { ) y de hospitalización especializada ( } 34 . \%) \text {. De estas quejas, 39.2\% demanda al IMSS y } \\
29.5 \% \text { al sector privado. El } 52 \% \text { de estas personas pertenece a la clase media baja y } 31 \% \text { es de clase media alta. }\end{array}$ \\
\hline 3 & $\begin{array}{c}325 \\
(16.88 \%)\end{array}$ & $\begin{array}{l}\text { Personas de la tercera edad } \\
\text { El promedio de edad de este grupo es de } 72 \text { años, } 56 \% \text { son mujeres y } 44 \% \text { son hombres. Las quejas involucran } \\
\text { principalmente al IMSS ( } 46 \% \text { ) y al ISSSTE (25\%). Las demandas se relacionan con el tratamiento ( } 39 . \%) \text {, y con las } \\
\text { intervenciones quirúrgicas ( } 26.4 \%) \text {. El } 43 \% \text { de los casos está vinculado a unidades de hospitalización especializada. El } \\
41 \% \text { pertenece a la clase media baja y } 37 \% \text { es de clase media alta. }\end{array}$ \\
\hline 4 & $\begin{array}{c}551 \\
(28.62 \%)\end{array}$ & $\begin{array}{l}\text { Adultos maduros } \\
\text { Personas que en promedio tienen } 51 \text { años, principalmente se trata de mujeres (61\%). Las quejas involucran al IMSS } \\
(39.3 \%) \text {, al ISSSTE ( } 28 \% \text { ) y al sector privado ( } 26 \%) \text {. El } 39 \% \text { de las demandas se refiere al tratamiento y } 28 \% \text { a } \\
\text { intervenciones quirúrgicas. Las unidades de hospitalización especializada son citadas en } 42 \% \text { de los casos. El } 45 \% \text { es } \\
\text { de clase media baja y } 31 \% \text { de clase media alta, } 10 \% \text { es el grupo con más personas de muy buena posición económica. }\end{array}$ \\
\hline
\end{tabular}


Los usuarios de la seguridad social reportan fundamentalmente insatisfacción con el diagnóstico, problemas de tipo administrativo y también se quejan de la relación médico-paciente, lo cual corrobora lo hallado por Aguirre ${ }^{34}$ en unidades del IMSS. Dentro de los problemas administrativos que identificamos, los más frecuentes se relacionan con las fechas de las citas para consultas e intervenciones quirúrgicas, que no se ajustan a las necesidades de los usuarios. El trabajo de Zurita, Nigenda y Ramírez ${ }^{35}$ describe la insatisfacción por las prolongadas esperas en las unidades del seguro social, que contrastan con la agilidad del sector privado.

Entre la población derechohabiente llama la atención el número de casos que informan haber acudido a buscar atención en el sector privado. En sí mismo, creemos que esto es un indicador de insatisfacción con la calidad de la atención ofrecida. En un estudio anterior realizado en México ${ }^{35}$ se halló que $40 \%$ de la población de la capital considera a los servicios privados como los mejores, y entre los asegurados del ISSSTE, 53\% pensaba de esa forma.

Las dificultades identificadas en las instituciones que atienden a la población no asegurada se caracterizan principalmente por problemas en la relación médico-paciente y en las intervenciones quirúrgicas. Si se considera que esta población usuaria por lo general es de escasos recursos, las 143 quejas presentadas, más que tomarse como un número relativamente pequeño, probablemente reflejan la falta de crítica de quienes se saben sin otras alternativas de atención.

Nuestros resultados muestran que en las instituciones de seguridad social, y en las que atienden a la población no asegurada, las quejas más frecuentes se refieren a problemas de estructura y del proceso de atención. Esto es consistente con un estudio previo de Ramirez, Nájera y Nigenda, ${ }^{33}$ donde se encontró que los principales motivos que la población señaló para no regresar a esos servicios fueron maltratos y pérdida de tiempo, así como la falta de medicamentos y material necesario. ${ }^{6}$

Las quejas que involucran a los servicios privados mayormente se refieren a insatisfacción con el tratamiento y con las intervenciones quirúrgicas. Se identificó que fundamentalmente se trata de inconformidades con los resultados de la atención, lo cual no contradice otros estudios previos..$^{33}$ Especulamos que como los costos de la atención privada son altos, en los usuarios se crean expectativas elevadas que los llevan a ser exigentes con los resultados esperados.

En nuestro estudio las mujeres presentaron la mayor cantidad de quejas, pero en la bibliografía revisada se registra que los resultados de la satisfacción asociada con el sexo son inconsistentes. ${ }^{2}$ En las quejas de las mujeres destaca que $22 \%$ se origina en los servicios de planificación familiar y ginecología, y fundamentalmente las quejas se refieren al tratamiento prescrito y a las intervenciones quirúrgicas. En el caso de los hombres, las quejas relacionadas con diagnósticos insatisfactorios es lo sobresaliente, particularmente en los servicios de traumatología y ortopedia.

Si en estudios anteriores ${ }^{2}$ se ha encontrado que las personas de mayor edad se muestran más satisfechas, nuestra información no nos permite comprobarlo. Sólo encontramos diferencias, mencionadas en los resultados, entre los tipos de quejas de menores y adultos. No se halló un patrón claro que diferencie las quejas presentadas según clases socioeconómicas. Tampoco podemos referirnos a la ocupación y la escolaridad, ya que la Conamed no recogió el dato inicialmente. Otros trabajos reportan que las personas de más instrucción son más críticas de la calidad y por lo tanto se muestran más insatisfechas. ${ }^{2,33,36}$ Sería conveniente y útil que en la Comisión recojan tales datos en el futuro.

En otros estudios sobre satisfacción de los pacientes hechos en otras regiones, ${ }^{17,37}$ al evaluar la calidad de la atención recibida los pacientes interrogados se refirieron con mayor frecuencia a cuestiones de tipo afectivo, como la comunicación y la relación interpersonal con el médico. Entre los casos que acuden a la Conamed es baja la frecuencia de quejas referidas a la relación médico-paciente como problema principal, aunque sí se observan elementos de ese tipo al revisar la narración de los hechos de la mayoría de las quejas llevadas ante la Comisión.

En esta discusión debemos señalar que los derechohabientes del IMSS y el ISSSTE antes de plantear una queja en la Conamed deben agotar los recursos que le ofrecen las propias instituciones involucradas. Lo anterior es en virtud de convenios de cooperación $^{38,39}$ firmados entre esas instituciones. Eso pudiera reducir en los próximos años el número de casos que procedentes de la seguridad social llegan a la Comisión y, derivado de lo anterior, aumentar la proporción de casos del sector privado, sin que necesariamente esa disminución indique que los problemas se han reducido en las instituciones de seguridad social. Este efecto aún no se nota en nuestros datos ya que se refieren a los dos primeros años de la Conamed.

La manera como se recogió la información que analizamos es diferente a la forma empleada en otros estudios sobre satisfacción con la atención o con la calidad de los servicios. Recordemos que las quejas que llegan a la Conamed son presentadas muchas veces en un momento aún reciente del problema, o en medio de éste, por lo que la mayoría de las veces están orientadas hacia las consecuencias directas sobre la salud y 
eso pudiera estar explicando por qué tantas quejas se relacionan directamente con el proceso de atención. Se trata de una situación diferente a cuando se pregunta sobre la satisfacción en general, o bien sobre aspectos concretos, sin que el informante esté involucrado en ese momento en una situación crítica.

A pesar de lo anterior, en general, nuestros resultados son consistentes con lo hallado en otros estudios sobre satisfacción y calidad percibida por usuarios de servicios de salud en México, por lo tanto, más allá de la forma en que se recoge la información, subsisten problemas reales en la atención médica que se presta. Constatamos que a través de una forma no convencional de estudiar la insatisfacción de los usuarios, como indicador de la calidad en los servicios médicos, también se puede obtener información útil para tomar decisiones con el fin de prevenir y corregir problemas futuros. Además, estudios como éste contribuyen con un conocimiento válido para plantear hipótesis de investigaciones futuras.

El número relativamente bajo de quejas recibidas en dos años en una ciudad de más de 8 millones de habitantes es uno de los resultados de este trabajo. A ello, estamos casi seguros que contribuyen dos factores, el primero es la escasa promoción hecha a una instancia como la Conamed y de las posibilidades que ofrece. Este factor se vuelve decisivo cuando se combina con otro: la pobre cultura de la población para enfrentar a quienes tradicionalmente han sido figuras de poder, los médicos. Cuando aquí usamos el término enfrentar no estamos abogando por fomentar una relación tensa entre los usuarios de servicios médicos y los prestadores, pero sí por una relación menos vertical y de mayor corresponsabilidad entre las partes involucradas, e indiscutiblemente, cuando una opción como la Conamed sea promovida, contribuiría notablemente a ello.

Los resultados aquí mostrados se derivan de una investigación más amplia ${ }^{29}$ que combinó métodos cualitativos, como el análisis de contenido y la profundización en el estudio de casos particulares, que permitieron sistematizar la información para su posterior manejo cuantitativo y por ello se pudo aprovechar una información que, por su espontaneidad, aporta datos más ricos que los ofrecidos por un instrumento preconcebido.

Esfuerzos futuros, que pudieran continuar la línea de investigación que aquí desarrollamos, serán muy valiosos, ya que sirven para ordenar lo que a primera vista pueden parecer problemas aislados que sucedieron a personas independientes, pero que realmente reflejan problemas recurrentes que caracterizan la calidad de los servicios médicos y las diversas formas que éstos tienen de manifestarse.

\section{Agradecimientos}

Los autores, especialmente el autor principal, reconocen la valiosa cooperación de la Dra. Rosario Cárdenas y el Dr. Juan Guillermo Figueroa en la realización de la Tesis $^{29}$ de la cual se derivan los resultados aquí mostrados. También expreso mi reconocimiento a la Secretaría de Relaciones Exteriores de México que otorgó la beca para cursar los estudios de Maestría y a la Organización Panamericana de la Salud que apoyó económicamente la realización de esta investigación.

\section{Referencias}

1. Larousse: El Pequeño Larousse llustrado 1999. Q uinta edición. México, D.F.: Ediciones Larousse, 1998:840.

2. Rees-Lewis J. Patient views on quality care in general practice: Literature review. Soc Sci Med 1994;39(5):655-670.

3. Fitzpatrick R. Surveys of patient satisfaction: Important general considerations. Br Med J 1991;302:887-889.

4.W illiams B. Patient satisfaction:A valid concept? Soc Sci Med 1994;38: 509-516.

5. D onabedian A. Garantía y monitoría de la calidad de la atención médica. Un texto introductorio. México, D.F.: Instituto $\mathrm{N}$ acional de Salud Pública, 1990; Perspectivas en Salud Pública 10-12

6. D onabedian A. Evaluating the quality of medical care. Milbank Memorial Fund Q 1966;44:429-462.

7. D onabedian A. La calidad de la atención médica. Definición y métodos de evaluación. México, D.F.: Ediciones científicas La Prensa Médica Mexicana, 1984.

8. Hall JA, Stein TS, Roter D, Rieser LN . Inaccuracies in physicians' perceptions of their patients. Soc Sci Med 1999;37(11):1164-1168.

9. Pfeffer $N$, C ooteA. Is quality good for you? So cial Policy Paper 5. Londres: Institute for Public Research, 1991.

10. Taylor A, Hudson K, Keeling A. Q uality nursing care, the costumer perspective revisited. J N urs Q ual Assur 1991;5(2):23-31.

11. D onabedian A. Continuidad y cambio en la búsqueda de la calidad. Salud Publica Mex 1993;35(3):238-247.

12. Stocker $\mathrm{K}, \mathrm{W}$ aitzkin $\mathrm{H}$, Iriart $\mathrm{C}$. The exportation of managed care to Latin A merica. N Engl J Med 199;340(14):1131-1136.

13. Holloway B, Mobbs D. A challenge we all can achieve. Professional N urse 1992:79-83.

14.W right S. Consuming interest. Senior N urse 1987;6(2):24-27.

15.A vis M. Incorporating patients' voices in the audit process. Q ual $H$ ealth Care 1997;6(2):86-91.

16. Hall JA, D orman MC.W hat patients like about their medical care and how often they asked:A meta analysis of satisfaction literarature. Soc Sci Med 1998;27:935-939.

17.W illiams SJ, Calnan M. Key determinant of consumer satisfaction with general practice. Fam Practice 1991;8:237-242.

18. Carr-Hill R, H umphreys K, Mclver S.A customised view of public satisfaction. Health Service J 1987;97:614-615.

19. Jung $H P, V a n$ H orne $F, W$ ensing $M, H$ earnshaw $H, G$ rol R.W hich aspects of general practitioners' behaviour determine patients' evaluations of care? Soc Sci Med 1998;47(8):1077-1087.

20. Calnan MW. The patient's perspective. Int J Technol Assess H ealth C are 1998;14(1):24-34. 
21. Comisión Nacional de Derechos Humanos, Informe Anual de Actividades. Mayo 1994 - Mayo 1995. México, D.F.: CN D 1995:547-551.

22. Comisión $\mathrm{N}$ acional de Derechos Humanos, El derecho a la protección de la salud de todos los mexicanos. Horacio García Romero (coord.) México, D.F.CNDH 1996a.

23. Comisión $N$ acional de Derechos Humanos, Informe Anual de Actividades. Mayo 1995 - Mayo 1996. México, D.F.CN DH 1996b:560-563.

24. Comisión Nacional de Derechos Humanos, Informe Anual de Actividades. Mayo 1996 - Mayo 1997. México, D.F.CN DH 1997:30-33.

25. Comisión Nacional de Derechos Humanos, Informe A nual de Actividades. Mayo 1997 - Mayo 1998. México, D.F.CN DH 1998:29-32.

26. Comisión $N$ acional de Arbitraje Médico: D ecreto de C reación. Decreto por el cual se crea la Comisión $\mathrm{N}$ acional de Arbitraje Médico. México, D.F.: Secretaría de Salud , 1996.

27. Fernández-Varela H. Conferencia Magistral “ $N$ aturaleza y misión de la Comisión N acional de Arbitraje Médico". I Simposio Internacional Por la Calidad de los Servicios Médicos y la Mejoría de la Relación MédicoPaciente. Convo cado por la Conamed y celebrado del 9 al 11 de diciembre de 1996 en México, D.F.

28.Valdés-Salgado R.A nálisis de las pretensiones que acompañan las quejas presentadas ante la Conamed. En: Rabell C, comp. Temas actuales de población. México, D.F.: Facultad Latinoamericana de Ciencias Sociales (Flacso) (en prensa).

29.Valdés-Salgado R. Satisfacción de los usuarios de servicios de salud. En: Valdés-Salgado R. Análisis de las demandas presentadas a la Conamed. Tesis para optar por el título de Maestro en Población. México, D.F.: Facultad Latinoamericana de Ciencias Sociales (Flacso), 1999.

30. Buró de Investigación de Mercados, Libro Mercadológico de la Megaciudad de México. México, D.F.: BIMSA 1998.
31. Sirkim RM. Statistics for the Social Science. Sage Publication, 1995 345-382.

32. Johnson RA,W ichern DW .A pplied multivariate statistical analysis.Third edition. N ueva Jersey: Prentice Hall, 1992:573-627.

33. Ramírez T de J, N ájera P, N igenda G. Percepción de la calidad de la atención de los servicios de salud en México: perspectiva de los usuarios. Salud Publica Mex 1998;40(1):3-12.

34.A guirre H. Evaluación de la atención médica. Expectativas de pacientes y trabajadores. Salud Publica Mex 1990;32(2):170-180.

35. Zurita B, N igenda G, Ramírez T de J. Encuesta de satisfacción con los servicios de salud, 1994. En: Frenk J, ed. 0 bservatorio de la salud. N ecesidades, servicios, política. México, D.F.: Fundación Mexicana para la Salud, 1997.

36. Sitzia J,W ood N . Patient satisfaction:A review of issues and concepts. Soc Sci Med 1997;45(12):1829-1843.

37. W are JE, Snyder MK, W right R, Davies AR. D efining and measuring patient satisfaction with medical care. Eval Program Plann 1983;6:247263.

38. Comisión $\mathrm{N}$ acional de Arbitraje Médico. Bases de coordinación que celebran la Comisión N acional de Arbitraje Médico y el Instituto de Seguridad y Servicios Sociales de los Trabajadores del Estado, para la atención de quejas médicas por la prestación de servicios. México, D.F., 12 de noviembre de 1997.

39. Comisión Nacional de Arbitraje Médico. Bases de coordinación que celebran la Comisión $\mathrm{N}$ acional de Arbitraje Médico y el Instituto Mexicano del Seguro Social. México, D.F. 